\title{
Comparison of optical quality and distinct macular thickness in femtosecond laser- assisted versus phacoemulsification cataract surgery
}

Yong Wang ${ }^{1,2+}$, Jinling Zhang ${ }^{1+}$, Miaomiao Qin ${ }^{1}$, Jianguo Miao ${ }^{1}$, Wei Chen ${ }^{1}$, Yemeng Huang ${ }^{1}$, Jian $\mathrm{Wu}^{1}$, Yu Guan ${ }^{1}$ and Huaijin Guan ${ }^{1 *}$

\begin{abstract}
Background: Optical quality and macular thickness changing optical quality is rarely reported after femtosecond laser-assisted cataract surgery (FLACS). In current research, we evaluated optical quality recovery and distinct macular thickness changes after FLACS and phacoemulsification cataract surgery (PCS).

Methods: A total of 100 cataract patients (100 eyes) were included (50 eyes for the FLACS group and 50 eyes for the PCS group). Modulation transfer function (MTF), point spread function (PSF) and dysfunctional lens index (DLI) were measured by a ray-tracing aberrometer (iTrace). Uncorrected distance visual acuity (UDVA) and corrected distance visual acuity (CDVA) were also assessed pre-operation, 1 week and 1 month after surgery. The MTF values at spatial frequencies of 5, 10, 15, 20, 25 and 30 cycles/degree (c/d) were selected. We used optical coherence tomography (OCT) to assess the macular thickness of different regions pre-operatively and 1 month after the surgery.

Results: In PCS group, we found the statistically significant differences between pre-operation and post-operation in DLI $(p<0.0001)$, PSF (strehl ratio, SR) $(p=0.027)$ and MTF $(p=0.028)$, but not intraocular pressure (IOP) $(p=0.857)$. The differences between pre-operation and post-operation for DLI $(p=0.031)$, SR $(p=0.01)$ and IOP $(p=0.03)$, but not MTF ( $p=0.128)$ were also found in FLACS group. The differences were statistically significant when the spatial frequencies were at 5, 10 and 25 ( $p=0.013,0.031$ and 0.048$)$ between pre-operation and post-operation in PCS group but not FLACS group at 1 month. In PCS group, we found the differences between pre-operation and postoperation in nasal inter macular ring thickness (NIMRT) $(p=0.03)$, foveal volume (FV) $(p=0.034)$ and average retinal thickness (ART) ( $p=0.025)$ but not FLACS group at 1 month.
\end{abstract}

Conclusion: FLACS is safe that did not cause significant increase of macular thickness in current study. However, it also cannot produce better optical quality. In contrast, PCS can produce macular thickness changes, but better optical quality recovery. The slightly retinal change may not affect optical quality.

Keywords: Optical quality, Macular thickness, Femtosecond laser-assisted cataract surgery (FLACS), Phacoemulsification cataract surgery (PCS), Dysfunctional lens index (DLI), Point spread function (PSF), Modulation transfer function (MTF)

\footnotetext{
* Correspondence: guanhuaijineye@163.com

†Yong Wang and Jinling Zhang contributed equally to this work.

${ }^{1}$ Department of Ophthalmology, Affiliated Hospital of Nantong University, 20

Xisi Road, Nantong, Jiangsu, China

Full list of author information is available at the end of the article
}

(c) The Author(s). 2020 Open Access This article is distributed under the terms of the Creative Commons Attribution 4.0 International License (http://creativecommons.org/licenses/by/4.0/), which permits unrestricted use, distribution, and reproduction in any medium, provided you give appropriate credit to the original author(s) and the source, provide a link to the Creative Commons license, and indicate if changes were made. The Creative Commons Public Domain Dedication waiver (http://creativecommons.org/publicdomain/zero/1.0/) applies to the data made available in this article, unless otherwise stated. 


\section{Background}

Femtosecond laser-assisted cataract surgery (FLACS) has gained popularity in recent years. It is the new technology suggesting potential improvements in clinical and safety outcomes over conventional phacoemulsification cataract surgery (PCS) [1, 2]. Many studies have highlighted that little clinically meaningful benefit exists from femtosecond laser pretreatment to cataract surgery [3, 4]. Even conventional PCS had a higher rate of cystoid macular edema (CME) than FLACS, research showed FLACS did not yield better visual or refractive outcomes than PCS $[4,5]$. Our previously study have illustrated that FLACS did not result in macular thickness changes in cataract patients with myopia [6]. However, weather the impact of FLACS on optical quality and macular thickness changing optical quality is rarely reported.

Vision can be assessed in terms of visual acuity and optical quality. The optical quality refers to the evaluation of the optical beam from the cornea to the retina. The global point spread function analysis are objective measurements of this optical quality [7]. In order to correct vision after cataract surgery, it is important to evaluate not only visual acuity but also optical quality. More and more researches focus on the relationship between intraocular lens (IOL) implantation and optical quality recovery $[8,9]$. However, few studies have investigated the changes in or influences on optical quality if the retina itself is not normal after cataract surgery.

Researcher analyzed the effects of retinal change on optical quality in central serous chorioretinopathy patients and found retinal change affected optical quality [10]. Retinal change especially macular thickness might be a sub-threshold retinal injury after FLACS and warrants further study [11]. A large comparative cohort study identified a higher rate of CME after FLACS [4]. However, other studies have shown that FLACS does not appear to increase macular thickness or CME more than PCS [3, 12-14]. Our previously study have shown that FLACS is safe for cataract patients with myopia that did not change the macular thickness [6]. However, we did not focus on the relationship between optical quality and macular thickness changes in the research.

In recent years, increasing scholars want to provide good optical quality for patients after cataract surgery. It is important to assess optical quality after the surgery. Modulation transfer function (MTF) is an objective method to evaluate imaging quality for human optical systems [15]. We also used strehl ratio (SR) to evaluate optical quality that is ideal PSF to aberrated PSF. It is defined as the ratio between the MTF area of the eye and the diffraction-limited MTF area [16]. Therefore, we assessed optical quality with MTF and SR in the study. Dysfunctional lens index (DLI) is a term coined to describe the natural aging changes in the lens as a novel surgery decision-maker $[17,18]$. In current study, we evaluated compare the optical quality outcomes and distinct macular thickness changes in FLACS and PCS group. In addition, we also analyzed the MTF values at spatial frequencies of $5,10,15,20,25$ and 30 cycles/ degree.

\section{Methods}

This observational prospective cohort study reviewed 100 patient records of FLACS and PCS cases performed by a single surgeon at Affiliated Hospital of Nantong University from January 2018 to September 2018. The study approved by the institutional ethics committee of Affiliated Hospital of Nantong University and was performed according to the tenets of the Declaration of Helsinki. All patients were willing to volunteer for the research and signed a written informed consent. We excluded the patients with previous ocular surgery, trauma and known macular alteration and all patients were given a complete ophthalmologic evaluation before surgery as our previously study. Uncorrected distance visual acuity (UDVA) and corrected distance visual acuity (CDVA) with subjective refraction performed by an optometrist using an LCD visual acuity chart preoperatively and 1 month postoperatively. Emery-Little classification was used to define the nuclear hardness. The Lens Opacities Classification System III (LOCS III) was used to define the lens opacities. The basic demographic of the study participant were listed in the Additional file 1: Table S1.All FLACS were performed by the same surgeon (H.J.G.) as our previously described [6]. All PCS surgeries also were performed by the same surgeon (H.J.G.). Tobramycin and Dexamethasone Eye Drops (Tobradex, Alcon) were used four times daily after surgery until day 14. Tobramycin and Dexamethasone Eye Ointment (Tobradex, Alcon) was used one time a day after surgery for 30 days. Pranoprofen eye drops (Pranopulin, Senju Pharmaceutical Co., Ltd.) was used three times a day after surgery for 30 days.

\section{Ray-tracing Aberrometry}

A wavefront aberrometry scan was performed with a ray-tracing aberrometer (iTrace, Tracey Technologies, Houston, TX) preoperatively and at the final 1 month follow-up as previously described [19]. Using the iTrace, we measured the optical quality parameters (MTF and SR) of the eye and DLI. For the MTF values measured at spatial frequencies, we selected $5,10,15,20,25$ and 30 cycles/degree (c/d). Pupils were dilated with a 6-mm size before measurement.

\section{OCT measurements}

OCT measurements (Cirrus HD-OCT 4000; Carl Zeiss Meditec, Dublin, CA) were performed 1 day before surgery and post-operation at 1 month as our previously described [6]. 


\section{Statistical analysis}

A SPSS 18.0 software (SPSS Inc., Chicago, Illinois) was performed for statistical analyses. Data are expressed as the mean and standard deviation. One-way ANOVA was performed to make comparisons between multiple subgroups for different macular region thickness, MTF, SR and DLI between FLACS group and PCS group in preoperation or post-operation. A $p$ value less than 0.05 was considered statistically significant.

\section{Results}

The FLACS group comprised of 50 eyes of 50 patients with the mean age of $56.66 \pm 5.68$ years. The PCS group comprised of 50 eyes of 50 patients with the mean age of $61.33 \pm 7.52$ years (Table 1 ). Preoperative intraocular pressure (IOP), AL, DLI, SR, MTF, UDVA, CDVA and nuclear hardness showed no statistically significant between two groups. We also found FLACS group produce less cumulative dissipated energy (CDE) but not ultrasound time when compared with PCS group (Table 1).

The postoperative IOP $(p=0.53 ; p=0.956)$, SR $(p=$ $0.53 ; p=0.975)$ and MTF $(p=0.28 ; p=0.537)$ no statistically significant between two groups, except DLI ( $p=$ 0.015) at 1 month. For UDVA and CDVA, we detected no significance between the two groups postoperatively at 1 week or 1 month. In PCS group, there are different between pre-operation and post-operation at 1 week and 1 month in DLI $(p=0.012 ; p<0.0001), \operatorname{SR}(p=0.015$; $p=0.027)$ and MTF $(p=0.008 ; p=0.028)$, but not IOP $(p=0.643 ; p=0.857)$ (Table 1). In FLACS group, we found there are different between pre-operation and post-operation at 1 week or 1 month in DLI ( $p=0.014$; $p=0.001)$, SR $(p=0.027 ; p=0.003)$, but not $\operatorname{MTF}(p=$ $0.18 ; p=0.128)$ and IOP $(p=0.758 ; p=0.31)$ (Table 1).

MTF values at 5, 10, 15, 20, 25 and 30 cycles/degree (c/ d) spatial frequencies were obtained pre-operation, 1 week and 1 month post-operation by iTrace. The comparison of MTF values at the same spatial frequencies showed rarely statistically significant between two groups pre-operation, 1 week and 1 month post-operation (Table 2). In PCS group, we did not find any statistically significant at 1 week. We also did not find any statistically significant at 1 month for 15,20 and 30 cycles/degree (c/d) spatial frequencies, except 5,10 and $25 \mathrm{cpd}(p=0.013,0.031$ and 0.048 ) (Table 2, Fig. 1a-c). However, there are not any statistically significant between pre-operation, 1 week and 1 month postoperation in FLACS group (Table 2).

Table 3 shows the pre- and postoperative macular thickness values in the FLACS and PCS group. There are not statistically significant after surgery between the two groups. We did not find any difference between preoperation and post-operation in FLACS group. But in PCS group, there are different between pre-operation and post-operation in nasal inter macular ring thickness (NIMRT) $(p=0.03)$, foveal volume (FV) $(p=0.034)$ and average retinal thickness (ART) $(p=0.025)$ (Table 3, Fig. 1e-f) at 1 month.

Table 1 Comparison of PCS Group and FLACS Group at Preoperative, Postoperative, 1 week and Postoperative 1 month

\begin{tabular}{|c|c|c|c|c|c|c|c|c|c|c|c|c|c|}
\hline \multirow{3}{*}{$\begin{array}{l}\text { Demographic/ } \\
\text { Parameter }\end{array}$} & \multicolumn{3}{|l|}{ PCS Group } & \multirow[t]{3}{*}{$P a$} & \multirow[t]{3}{*}{$P b$} & \multicolumn{3}{|l|}{ FLACS Group } & \multirow[t]{3}{*}{$P C$} & \multirow[t]{3}{*}{$P d$} & \multirow[t]{3}{*}{$P e$} & \multirow[t]{3}{*}{$P f$} & \multirow[t]{3}{*}{$P g$} \\
\hline & Preoperative & Postoperative & Postoperative & & & \multirow[t]{2}{*}{ Preoperative } & Postoperative & Postoperative & & & & & \\
\hline & & 1 week & 1 month & & & & 1 week & 1 month & & & & & \\
\hline UCVA & $0.69 \pm 0.32$ & $0.16 \pm 0.23$ & $0.19 \pm 0.28$ & 0.002 & 0.003 & $0.86 \pm 0.44$ & $0.19 \pm 0.24$ & $0.21 \pm 0.17$ & 0.001 & 0.012 & 0.68 & 0.2 & 0.746 \\
\hline BCVA & $0.58 \pm 0.34$ & $0.18 \pm 0.14$ & $0.12 \pm 0.18$ & 0.005 & 0.001 & $0.74 \pm 0.49$ & $0.19 \pm 0.39$ & $0.19 \pm 0.28$ & 0.004 & 0.019 & 0.739 & 0.48 & 0.656 \\
\hline IOP & $15.75 \pm 2.5$ & $16.33 \pm 3.4$ & $15.72 \pm 2.15$ & 0.643 & 0.857 & $14.33 \pm 2.14$ & $15.23 \pm 3.39$ & $15.69 \pm 1.14$ & 0.758 & 0.031 & 0.056 & 0.53 & 0.956 \\
\hline DLI & $3.14 \pm 1.73$ & $6.14 \pm 2.33$ & $6.51 \pm 2.78$ & 0.012 & $<0.0001$ & $2.65 \pm 0.78$ & $4.25 \pm 0.68$ & $4.49 \pm 1.94$ & 0.014 & 0.001 & 0.198 & 0.37 & 0.015 \\
\hline SR & $\begin{array}{l}0.0066 \pm \\
0.0076\end{array}$ & $\begin{array}{l}0.0094 \pm \\
0.0055\end{array}$ & $0.012 \pm 0.009$ & 0.015 & 0.027 & $\begin{array}{l}0.0045 \pm \\
0.0023\end{array}$ & $\begin{array}{l}0.0115 \pm \\
0.0063\end{array}$ & $0.012 \pm 0.0086$ & 0.027 & 0.003 & 0.282 & 0.28 & 0.975 \\
\hline MTF & $0.11 \pm 0.07$ & $0.11 \pm 0.07$ & $0.17 \pm 0.1$ & 0.008 & 0.028 & $0.13 \pm 0.13$ & $0.18 \pm 0.14$ & $0.19 \pm 0.12$ & 0.018 & 0.128 & 0.636 & 0.34 & 0.537 \\
\hline Age (y) & $56.66 \pm 5.68$ & & & & & $61.33 \pm 7.52$ & & & & & 0.14 & & \\
\hline$A L$ & $23.28 \pm 1.64$ & & & & & $23.84 \pm 2.65$ & & & & & 0.378 & & \\
\hline phaco time & $14.59 \pm 7.68$ & & & & & $13.89 \pm 14.87$ & & & & & 0.839 & & \\
\hline CDE & $14.59 \pm 7.68$ & & & & & $2.24 \pm 2.79$ & & & & & $\begin{array}{l}< \\
0.0001\end{array}$ & & \\
\hline $\begin{array}{l}\text { nuclear } \\
\text { hardness }\end{array}$ & $2.11 \pm 0.33$ & & & & & $2.42 \pm 0.51$ & & & & & 0.15 & & \\
\hline
\end{tabular}

IOP intraocular pressure, $A L$ axial length, DLI Dysfunctional Lens Index, UCVA uncorrected viusal acuity, BCVA best corrected viusal acuity, SR strehl ratio, MTF modulation transfer function, CDE cumulative dissipated energy

$P$ a: Comparison of Preoperative and Postoperative 1 Week in PCS Group; $P b$ : Comparison of Preoperative and Postoperative 1 Month in PCS Group $P$ c: Comparison of Preoperative and Postoperative 1 Week in FLACS Group; $P d$ : Comparison of Preoperative and Postoperative 1 Month in FLACS Group

$P$ e: Comparison of PCS Group and FLACS Group at Preoperative; P f: Comparison of PCS Group and FLACS Group at Postoperative 1 Week

$P$ g: Comparison of PCS Group and FLACS Group at Postoperative 1 Month 
Table 2 Preoperative and Postoperative difference spatial frequencies MTF comparison for PCS group and FLACS group

\begin{tabular}{|c|c|c|c|c|c|c|c|c|c|c|c|c|c|}
\hline \multirow[t]{3}{*}{ Visit } & \multicolumn{3}{|l|}{ PCS Group } & \multirow[t]{3}{*}{$P a$} & \multirow[t]{3}{*}{$P b$} & \multicolumn{3}{|l|}{ FLACS Group } & \multirow[t]{3}{*}{$P C$} & \multirow[t]{3}{*}{$P d$} & \multirow[t]{3}{*}{$P e$} & \multirow[t]{3}{*}{$P f$} & \multirow[t]{3}{*}{$P g$} \\
\hline & \multirow[t]{2}{*}{ Preoperative } & Postoperative & Postoperative & & & \multirow[t]{2}{*}{ Preoperative } & \multirow[b]{2}{*}{1 week } & \multirow{2}{*}{$\begin{array}{l}\text { Postoperative } \\
1 \text { month }\end{array}$} & & & & & \\
\hline & & 1 week & 1 month & & & & & & & & & & \\
\hline $5 \mathrm{cpd}$ & $0.09 \pm 0.1$ & $0.19 \pm 0.19$ & $0.23 \pm 0.25$ & 0.096 & 0.013 & $0.19 \pm 0.24$ & $0.29 \pm 0.19$ & $0.37 \pm 0.26$ & 0.45 & 0.05 & 0.078 & 0.42 & 0.0 \\
\hline 10cpd & $0.02 \pm 0.037$ & $0.06 \pm 0.07$ & $0.07 \pm 0.12$ & 0.053 & 0.031 & $0.078 \pm 0.163$ & $0.13 \pm 0.2$ & $0.15 \pm 0.19$ & 0.225 & 0.264 & 0.111 & 0.364 & 0.1 \\
\hline $15 \mathrm{cpd}$ & $0.01 \pm 0.02$ & $0.063 \pm 0.19$ & $0.036 \pm 0.057$ & 0.48 & 0.051 & $0.04 \pm 0.1$ & $0.063 \pm 0.13$ & $0.085 \pm 0.129$ & 0.224 & 0.311 & 0.156 & 0.451 & 0.1 \\
\hline 20cpd & $0.07 \pm 0.14$ & $0.019 \pm 0.03$ & $0.022 \pm 0.034$ & 0.2 & 0.052 & $0.025 \pm 0.062$ & $0.04 \pm 0.092$ & $0.05 \pm 0.078$ & 0.285 & 0.321 & 0.164 & 0.332 & 0.1 \\
\hline $25 \mathrm{cpd}$ & $0.004 \pm 0.01$ & $0.017 \pm 0.019$ & $0.014 \pm 0.022$ & 0.21 & 0.048 & $0.017 \pm 0.0044$ & $0.026 \pm 0.64$ & $0.029 \pm 0.039$ & 0.177 & 0.405 & 0.181 & 0.532 & 0.1 \\
\hline $30 \mathrm{cpd}$ & $0.0036 \pm 0.0086$ & $0.087 \pm 0.014$ & $0.01 \pm 0.015$ & 0.19 & 0.06 & $0.0113 \pm 0.03$ & $0.03 \pm 0.484$ & $0.019 \pm 0.022$ & 0.324 & 0.422 & 0.23 & 0.332 & \\
\hline
\end{tabular}

cpd cycles per degree, MTF modulation transfer function

$P$ a: Comparison of Preoperative and Postoperative 1 Week in PCS Group; $P$ b: Comparison of Preoperative and Postoperative 1 Month in PCS Group

$P$ c: Comparison of Preoperative and Postoperative 1 Week in FLACS Group; $P$ d: Comparison of Preoperative and Postoperative 1 Month in FLACS Group

$P$ e: Comparison of PCS Group and FLACS Group at Preoperative; $P$ f: Comparison of PCS Group and FLACS Group at Postoperative 1 Week

$P$ g: Comparison of PCS Group and FLACS Group at Postoperative 1 Month

\section{Discussion}

Optical quality after cataract surgery gained more and more attention for providing satisfactory visual outcomes [20,21]. Studies have shown that FLACS produce better clear corneal incision morphology [8], more precise reproducible capsulotomies [9-13], and better IOL centration [11] when compared with conventional PCS. Even with these reported benefits, it still needs to be proven whether FLACS can produce better optical quality than conventional PCS. Cataract surgery can

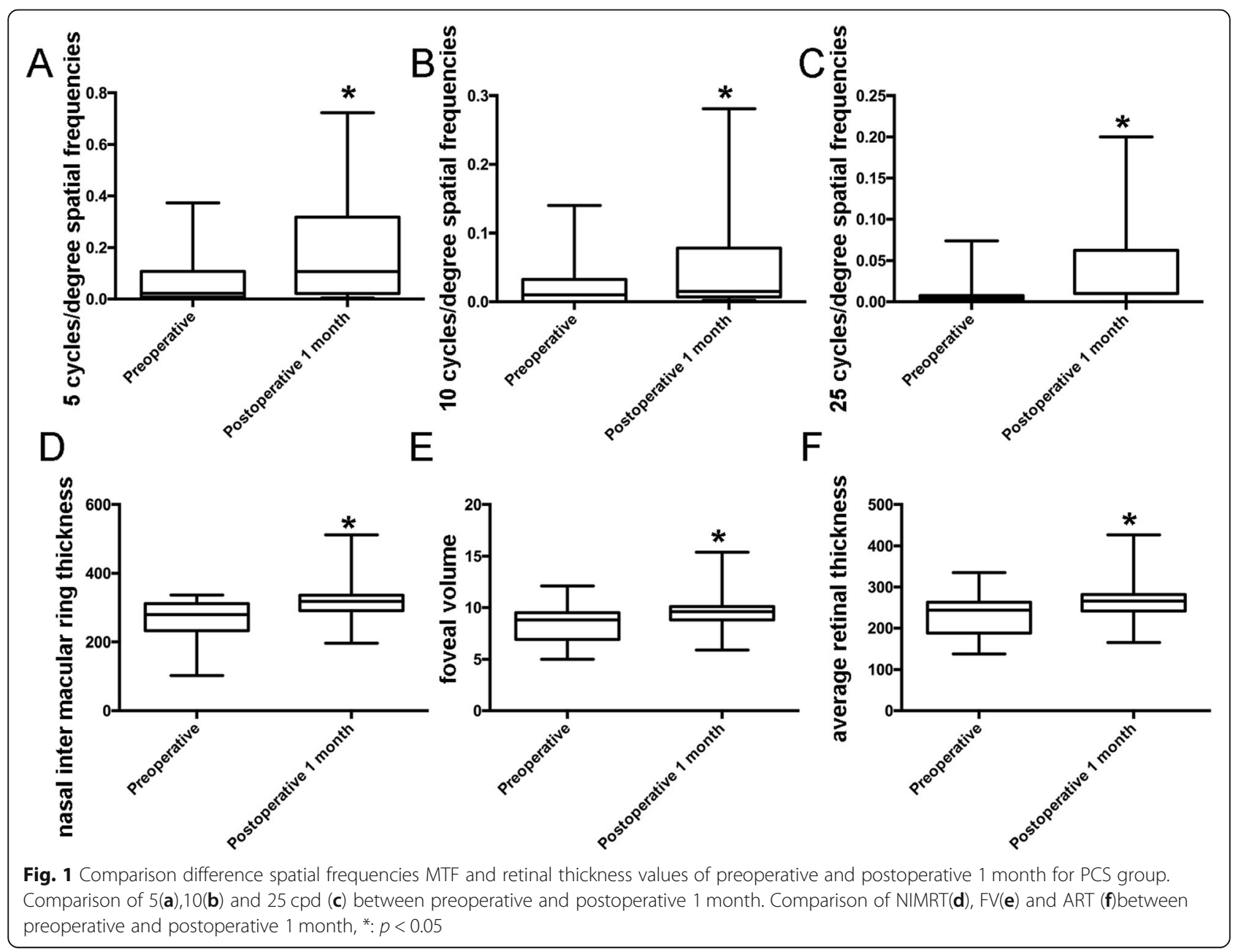


Table 3 Comparison of retinal thickness values of PCS group and FLACS group

\begin{tabular}{|c|c|c|c|c|c|c|c|c|c|c|c|c|c|}
\hline \multirow[t]{3}{*}{ Visit } & \multicolumn{3}{|l|}{ PCS Group } & \multirow[t]{3}{*}{$P a$} & \multirow[t]{3}{*}{$P b$} & \multicolumn{3}{|l|}{ FLACS Group } & \multirow[t]{3}{*}{$P C$} & \multirow[t]{3}{*}{$P d$} & \multirow[t]{3}{*}{$P e$} & \multirow[t]{3}{*}{$P f$} & \multirow[t]{3}{*}{$P g$} \\
\hline & \multirow[t]{2}{*}{ Preoperative } & Postoperative & Postoperative & & & \multirow[t]{2}{*}{ Preoperative } & \multirow{2}{*}{$\begin{array}{l}\text { Postoperative } \\
1 \text { week }\end{array}$} & \multirow{2}{*}{$\begin{array}{l}\text { Postoperative } \\
1 \text { month }\end{array}$} & & & & & \\
\hline & & 1 week & 1 month & & & & & & & & & & \\
\hline SOMRT & $272.33 \pm 51.69$ & $275.79 \pm 43.92$ & $289.85 \pm 81.45$ & 0.43 & 0.35 & $\begin{array}{l}272.33 \pm \\
51.69\end{array}$ & $278.94 \pm 62.93$ & $289.85 \pm 81.45$ & 0.469 & 0.923 & 0.476 & 0.651 & 0.207 \\
\hline IOMRT & $249.11 \pm 44.33$ & $259.79 \pm 61.32$ & $265.63 \pm 56.8$ & 0.221 & 0.239 & $\begin{array}{l}249.56 \pm \\
61.19\end{array}$ & $255.18 \pm 49.71$ & $260.17 \pm 49.89$ & 0.768 & 0.572 & 0.863 & 0.26 & 0.905 \\
\hline BOMRT & $204.19 \pm 96.7$ & $221 \pm 62.75$ & $230.59 \pm 95.68$ & 0.683 & 0.318 & $\begin{array}{l}208.67 \pm \\
61.86\end{array}$ & $239.64 \pm 34.18$ & $233.89 \pm 80.31$ & 0.078 & 0.299 & 0.978 & 0.279 & 0.742 \\
\hline NOMRT & $281.59 \pm 29.05$ & $275.63 \pm 24.87$ & $289.59 \pm 51.88$ & 0.472 & 0.488 & $\begin{array}{l}279.56 \pm \\
92.48\end{array}$ & $286.88 \pm 18.97$ & $294.89 \pm 89.86$ & 0.751 & 0.617 & 0.915 & 0.14 & 0.803 \\
\hline SIMRT & $\begin{array}{l}249.267 \pm \\
67.31\end{array}$ & $299 \pm 82.42$ & $\begin{array}{l}265.37 \pm \\
106.61\end{array}$ & 0.345 & 0.508 & $\begin{array}{l}276.94 \pm \\
57.25\end{array}$ & $284.88 \pm 38.93$ & $288.11 \pm 74.45$ & 0.289 & 0.617 & 0.159 & 0.852 & 0.435 \\
\hline BIMRT & $239.89 \pm 84.46$ & $276.15 \pm 103$ & $\begin{array}{l}284.41 \pm \\
105.31\end{array}$ & 0.197 & 0.093 & $227 \pm 77.54$ & $263 \pm 62.17$ & $\begin{array}{l}256.831 \pm \\
87.49\end{array}$ & 0.141 & 0.287 & 0.607 & 0.65 & 0.363 \\
\hline IIMRT & $253.81 \pm 66.27$ & $282.79 \pm 91.58$ & $282.67 \pm 99.6$ & 0.219 & 0.216 & $\begin{array}{l}243.94 \pm \\
93.51\end{array}$ & $280.23 \pm 47.39$ & $288.5 \pm 36.95$ & 0.161 & 0.069 & 0.68 & 0.918 & 0.814 \\
\hline NIMRT & $260.4 \pm 70.71$ & $295.31 \pm 71.67$ & $305.15 \pm 76.13$ & 0.108 & 0.03 & $\begin{array}{l}284.33 \pm \\
85.79\end{array}$ & $295.11 \pm 32.6$ & $302.27 \pm 54.77$ & 0.63 & 0.46 & 0.313 & 0.992 & 0.891 \\
\hline FT & $152.85 \pm 83.28$ & $202 \pm 53.31$ & $\begin{array}{l}187.52 \pm \\
120.01\end{array}$ & 0.06 & 0.223 & $\begin{array}{l}171.11 \pm \\
78.49\end{array}$ & $203.64 \pm 85.38$ & $\begin{array}{l}233.11 \pm \\
114.03\end{array}$ & 0.249 & 0.66 & 0.465 & 0.442 & 0.21 \\
\hline FV & $8.29 \pm 1.73$ & $8.58 \pm 1.78$ & $9.37 \pm 1.92$ & 0.578 & 0.034 & $8.39 \pm 1.66$ & $8.9 \pm 1.26$ & $9.06 \pm 1.93$ & 0.319 & 0.277 & 0.834 & 0.542 & 0.598 \\
\hline ART & $228.3 \pm 48.52$ & $231 \pm 69.61$ & $260.19 \pm 53.22$ & 0.877 & 0.025 & $\begin{array}{l}233.67 \pm \\
45.66\end{array}$ & $247.17 \pm 34.98$ & $251.22 \pm 53.37$ & 0.318 & 0.283 & 0.737 & 0.76 & 0.583 \\
\hline
\end{tabular}

SOMRT superior outer macular ring thickness, BOMRT bitamporal outer macular ring thickness, IOMRT inferior outer macular ring thickness, NOMRT nasal outer macular ring thickness, SIMRT superior inter macular ring thickness, BIMRT bitamporal inter macular ring thickness, IIMRT inferior inter macular ring thickness, NIMRT nasal inter macular ring thickness, FT foveal thickness, FV foveal volume, ART average retinal thickness

$P$ a: Comparison of Preoperative and Postoperative 1 Week in PCS Group; $P$ : Comparison of Preoperative and Postoperative 1 Month in PCS Group $P$ c: Comparison of Preoperative and Postoperative 1 Week in FLACS Group; $P$ d: Comparison of Preoperative and Postoperative 1 Month in FLACS Group $P$ e: Comparison of PCS Group and FLACS Group at Preoperative; $P f$ : Comparison of PCS Group and FLACS Group at Postoperative 1 Week

$P g$ : Comparison of PCS Group and FLACS Group at Postoperative 1 Month

cause macular thickness change. The complication has brought substantial attention to surgeons due to its potential hazard to vision consequence [14, 22]. However, the complication changed optical quality still needs to be investigated. Therefore, we studied optical quality by MTF, SR and distinct macular thickness for the two groups in current study.

In our study, the UCDA and CDVA shown no significance between the two groups preoperatively and postoperatively, the results were consisted with previously studies that FLACS did not yield better visual results [4, 23]. Studies have shown that FLACS produce significant reduction in effective phacoemulsification time [23, 24], reduce ultrasound power and ultrasound time [25]. In the research, our results show that FLACS can reduce CDE but not phacoemulsification time. We speculate the reason is that FLACS can pre-chop the lens nuclear. Elevation or rapid fluctuations in IOP may cause vascular or rhegmatogenous events [26]. In FLACS group, we found postoperative IOP were raised, the results are consisted with previous researches [27, 28]. Researches have shown that cytokines in anterior chamber after FLACS are higher than conventional PSC [29]. Therefore, we hypothesize that the raised
IOP may be associated with these cytokines which leading to increased resistance at the trabecular meshwork. Whether the raised IOP after surgery affects the retinal nerve fiber layer (RNFL) and visual field which need longterm follow-up and further research.

To better understand optical quality after FLACS, the MTF and SR were measured. MTF is the ratio between the image contrast of a specific object through the imaging optical system and the contrast of the object itself [15]. In general, the higher the MTF and SR, the better the ocular optical quality. In PCS group, we found there are different between pre-operation and post-operation in SR and MTF. Furthermore, the differences are statistically significant when the spatial frequencies are at 5, 10 and 25 cycles/degree (c/d). In FLACS group, we found there are different between pre-operation and post-operation in SR but not MTF. We speculate whether FLACS increase surgery induced astigmatism (SIA) compared with PCS which result in not difference between pre-operation and post-operation for MTF [30]. Even though, MTF value is undoubtedly an objective and accurate indicator for optical quality evaluation [31], the assessment of optical quality cannot be completed by one single indicator. 
Other objective and subjective indicators should also be integrated to make an accurate assessment. Therefore, we measured DLI in the two groups. The DLI in PCS group is significantly higher than the FLACS group in the baseline measurement (post-operative).

Then, we future analyzed the preoperative and postoperative macular thickness values in PCS and FLACS group by OCT. CME can be detected at the first week and peaks about 4 weeks after surgery [32]. In our study, macular thicknesses were performed before surgery and post-operation at 1 month. In PCS group, there are different between pre-operation and post-operation in NIMRT, FV and ART, but not in FLACS group. The results are consisted with previous research that the FLACS does not difference in postoperative macular thickness as compared with PCS [32].

In this study, the limitation includes a relatively small number of patients and short follow-up period. The other limitation is that the eyes were not randomized for FLACS group or PCS group. The current results might have selection bias, although there are no different in nuclear density between two groups. The comparison of optical quality between FLACS group and PCS group needs a long-term follow-up and further research. Such as capsular fibrosis and posterior capsular opacification (PCO) may cause optical quality change in the two groups. Whether the increasing IOP after FLACS has an effect on the RNFL also requires further investigation.

\section{Conclusions}

Our results suggest although FLACS did not result in macular thickness change compare with PCS, it also cannot gain better optical quality recovery at 1 month after surgery. The slightly retinal change may not affect optical quality in PCS group.

\section{Supplementary information}

Supplementary information accompanies this paper at https://doi.org/10. 1186/s12886-020-1319-3

Additional file 1: Supplementary Table.

\footnotetext{
Abbreviations

ART: Average retinal thickness; BIMRT: Bitamporal inter macular ring thickness; BOMRT: Bitamporal outer macular ring thickness; CDVA: Corrected distance visual acuity; CME: Cystoid macular edema; cpd: Cycles per degree; DLI: Dysfunctional lens index; FLACS: Femtosecond laser-assisted cataract surgery; FT: Foveal thickness; FV: Foveal volume; IIMRT: Inferior inter macular ring thickness; IOL: Intraocular lens; MTF: Modulation transfer function; NIMRT: Nasal inter macular ring thickness; NOMRT: Nasal outer macular ring thickness; OCT: Optical coherence tomography; OMRT: Inferior outer macular ring thickness; PCO: Posterior capsular opacification; PCS: Phacoemulsification cataract surgery; PSF: Point spread function; SIMRT: Superior inter macular ring thickness; SOMRT: Superior outer macular ring thickness;

UDVA: Uncorrected distance visual acuity
}

\section{Authors' contributions}

YW and JLZ performed the experiments. MMQ, WC, YMH, JGM and YG participated in data analysis. YW, JLZ, JW and JY wrote the manuscript. HJG conceived the research, and critically reviewed the manuscript and interpreted the data. All authors read and approved the final manuscript.

\section{Funding}

This study was supported by the National Natural Science Foundation of China (No. 81873676 and No. 81770906) and Science and technology for people's livelihood of Nantong (MS22019012).

\section{Availability of data and materials \\ The datasets used during the current study are available from the corresponding author on reasonable request.}

\section{Ethics approval and consent to participate}

The study approved by the institutional ethics committee of Affiliated Hospital of Nantong University and was performed according to the tenets of the Declaration of Helsinki. All patients were willing to volunteer for the research and signed a written informed consent.

Consent for publication

Not applicable

\section{Competing interests}

The authors declare that they have no competing interests.

\section{Author details}

${ }^{1}$ Department of Ophthalmology, Affiliated Hospital of Nantong University, 20 Xisi Road, Nantong, Jiangsu, China. ${ }^{2}$ Nantong University, Nantong, Jiangsu, China.

Received: 3 September 2019 Accepted: 17 January 2020

Published online: 01 February 2020

\section{References}

1. Gupta PC, Ram J. Femtosecond laser-assisted cataract surgery in complex cases. J Cataract Refract Surg. 2016;42(11):1693.

2. Agarwal A, Jacob S. Current and effective advantages of femto phacoemulsification. Curr Opin Ophthalmol. 2017;28(1):49-57.

3. Manning $S$, et al. Femtosecond laser-assisted cataract surgery versus standard phacoemulsification cataract surgery: study from the European registry of quality outcomes for cataract and refractive surgery. J Cataract Refract Surg. 2016:42(12):1779-90.

4. Ewe SY, et al. A comparative cohort study of visual outcomes in femtosecond laser-assisted versus phacoemulsification cataract surgery. Ophthalmology. 2016;123(1):178-82.

5. Popovic M, et al. Efficacy and safety of femtosecond laser-assisted cataract surgery compared with manual cataract surgery: a meta-analysis of 14567 eyes. Ophthalmology. 2016;123(10):2113-26.

6. Wang $Y$, et al. Distinct macular thickness changes after femtosecond laserassisted cataract surgery of age-related cataract and myopia with cataract. Sci Rep. 2018:8(1):3279.

7. Donate $D$, Thaeron R. SMILE with low energy levels: assessment of early visual and optical quality recovery. J Refract Surg. 2019:35(5):285-93.

8. Liao, X., et al., Comprehensive Evaluation of Retinal Image Quality in Comparing Different Aspheric to Spherical Intraocular Lens Implants. Curr Eye Res. 2019;44(10):1098-1103

9. Trindade BLC, et al. Versatile optical setup customized to verify the quality of spherical and aspheric intraocular lenses. J Opt Soc Am A Opt Image Sci Vis. 2019;36(4):B132-b137.

10. Lee K, et al. Optical quality in central serous chorioretinopathy. Invest Ophthalmol Vis Sci. 2014;55(12):8598-603.

11. Ewe SY, et al. Cystoid macular edema after femtosecond laser-assisted versus phacoemulsification cataract surgery. J Cataract Refract Surg. 2015; 41(11):2373-8

12. Levitz $L$, et al. Incidence of cystoid macular edema: femtosecond laserassisted cataract surgery versus manual cataract surgery. J Cataract Refract Surg. 2015;41(3):683-6. 
13. Conrad-Hengerer l, et al. Effect of femtosecond laser fragmentation on effective phacoemulsification time in cataract surgery. J Refract Surg. 2012; 28(12):879-83.

14. Conrad-Hengerer I, et al. Femtosecond laser-induced macular changes and anterior segment inflammation in cataract surgery. J Refract Surg. 2014; 30(4):222-6.

15. Kawamorita T, Uozato H. Modulation transfer function and pupil size in multifocal and monofocal intraocular lenses in vitro. J Cataract Refract Surg. 2005;31(12):2379-85.

16. Mafusire C, Kruger TP. Strehl ratio and amplitude-weighted generalized orthonormal Zernike-based polynomials. Appl Opt. 2017;56(8):2336-45.

17. Li Z, et al. Dysfunctional Lens index serves as a novel surgery decisionmaker for age-related nuclear cataracts. Curr Eye Res. 2019;44(7):733-8.

18. Kamiya $\mathrm{K}$, et al. Time course of optical quality and intraocular scattering after refractive lenticule extraction. PLoS One. 2013:8(10):e76738.

19. Srivastava $\mathrm{S}$, et al. Comparison of ocular wavefront aberrations in subluxated lenses before and after lens extraction with intraocular lens implantation. J Cataract Refract Surg. 2018;44(3):336-40.

20. Jimenez R, et al. Optical quality and visual performance after cataract surgery with biaxial microincision intraocular lens implantation. J Cataract Refract Surg. 2016;42(7):1022-8.

21. Hwang JS, et al. Utility of the optical quality analysis system for decisionmaking in cataract surgery. BMC Ophthalmol. 2018;18(1):231.

22. Stifter $\mathrm{E}$, et al. Macular morphology after cataract surgery with primary posterior capsulorhexis and posterior optic buttonholing. Am J Ophthalmol. 2008;146(1):15-22

23. Reddy KP, Kandulla J, Auffarth GU. Effectiveness and safety of femtosecond laser-assisted lens fragmentation and anterior capsulotomy versus the manual technique in cataract surgery. J Cataract Refract Surg. 2013;39(9): 1297-306.

24. Abell RG, Kerr NM, Vote BJ. Toward zero effective phacoemulsification time using femtosecond laser pretreatment. Ophthalmology. 2013;120(5):942-8.

25. Daya SM, Nanavaty MA, Espinosa-Lagana MM. Translenticular hydrodissection, lens fragmentation, and influence on ultrasound power in femtosecond laser-assisted cataract surgery and refractive lens exchange. Cataract Refract Surg. 2014;40(1):37-43.

26. He L, Sheehy K, Culbertson W. Femtosecond laser-assisted cataract surgery. Curr Opin Ophthalmol. 2011;22(1):43-52.

27. Chee SP, Yang Y, Ti SE. Clinical outcomes in the first two years of femtosecond laser-assisted cataract surgery. Am J Ophthalmol. 2015; 159(4):714-9.

28. Kerr NM, et al. Intraocular pressure during femtosecond laser pretreatment of cataract. J Cataract Refract Surg. 2013;39(3):339-42.

29. Kiss $\mathrm{HJ}$, et al. One-day use of preoperative topical nonsteroidal antiinflammatory drug prevents intraoperative prostaglandin level elevation during femtosecond laser-assisted cataract surgery. Curr Eye Res. 2016:41(8):1064-7.

30. Zhu S, et al. Morphologic features and surgically induced astigmatism of femtosecond laser versus manual clear corneal incisions. J Cataract Refract Surg. 2017;43(11):1430-5

31. Zheng $Y Z$, et al. Analysis of the optical quality by determining the modulation transfer function for anterior corneal surface in myopes. Int J Ophthalmol. 2012;5(2):196-201.

32. Ecsedy $M$, et al. Effect of femtosecond laser cataract surgery on the macula. J Refract Surg. 2011;27(10):717-22.

\section{Publisher's Note}

Springer Nature remains neutral with regard to jurisdictional claims in published maps and institutional affiliations.

Ready to submit your research? Choose BMC and benefit from:

- fast, convenient online submission

- thorough peer review by experienced researchers in your field

- rapid publication on acceptance

- support for research data, including large and complex data types

- gold Open Access which fosters wider collaboration and increased citations

- maximum visibility for your research: over $100 \mathrm{M}$ website views per year

At $\mathrm{BMC}$, research is always in progress.

Learn more biomedcentral.com/submissions 